\title{
Can we develop a Canadian Perioperative Anesthesiology Clinical Trials Group?
}

\author{
Richard Hall, MD $\cdot$ Scott Beattie, MD $\cdot$ Davy Cheng, MD $\cdot$ Peter Choi, MD · \\ André Y. Denault, MD - David Mazer, MD - W. Alan C. Mutch, MD • \\ Alexis F. Turgeon, MD • Homer Yang, MD
}

Received: 9 August 2010/Accepted: 30 August 2010/Published online: 28 September 2010

(c) Canadian Anesthesiologists' Society 2010

\section{The development of a collaborative research network in Canada: what is PACT?}

In Canada, research in the field of anesthesiology has been restricted mainly to the domains of basic science and applied physiology. Despite being of valuable scientific relevance and importance, most of this research evaluates physiologic outcomes or surrogate clinical outcomes rather than clinically significant outcomes that could change the way in which we practice. While many medical specialties have appreciated the importance of evaluating outcomes, such as mortality, quality of life, length of stay or efficiency of care, anesthesiology has lagged behind. One of the main reasons Canadian anesthesiology has been slow to embrace outcome studies is the delayed development of a collaborative network of anesthesia investigators to advance multicentre clinical research projects. Clinical research is at a turning point and is rapidly evolving, not only in Canada but worldwide. We strongly believe that

R. Hall, MD · S. Beattie, MD - D. Cheng, MD · P. Choi, MD .

A. Y. Denault, MD · D. Mazer, MD - W.

A. C. Mutch, MD - A. F. Turgeon, MD - H. Yang, MD

Perioperative Anesthesiology Clinical Trials Group Steering

Committee, Halifax, NS, Canada

R. Hall, MD ( $\square)$

Departments of Anesthesiology, Medicine and Pharmacology, Dalhousie University and the Capital District Health Authority, Rm 5452-Halifax Infirmary, 1796 Summer Street, Halifax, NS B3H 3A7, Canada

e-mail: rihall@dal.ca
Canadian anesthesiologists are capable of markedly improving their research capacity by fostering collaborative outcome-driven research. We further believe that such research can and will change clinical practice. In order to attain this goal, we have initiated a collaborative research network, the Perioperative Anesthesiology Clinical Trials (PACT) group.

The PACT group is a collaborative group of Canadian academic anesthesiologists with an interest in the design, implementation, conduct, and publication of multicentre clinical trials in anesthesiology and perioperative medicine. The aim of the PACT initiative is to help anesthesiology clinician investigators 1) to identify and investigate clinically relevant questions in anesthesiology and perioperative medicine; 2) to build research programs that answer their research questions; 3 ) to structure and conduct quality research with sound methodology; 4) to facilitate collaboration amongst various investigators across Canada so as to conduct multicentre research; 5) to acquire peer-reviewed funding from granting agencies; and finally 6) to execute and publish their results in high-impact peer-reviewed journals. The primary focus of the PACT initiative is to generate knowledge in perioperative anesthesia by providing a forum for the development and implementation of multicentre clinical trials that are designed to answer research questions. It is anticipated that the forum will have a fundamental impact on the practice of anesthesiology and perioperative medicine. In addition, the PACT group will provide mentoring to new investigators as a collaborative network and will facilitate the dissemination of research knowledge, best practices, and standardization of practice parameters, thereby informing the practice of anesthesiology and ensuring its continued advancement as a profession. 
Why do we need the PACT initiative, and what are the benefits of having my program become a PACTsponsored research program?

The research environment in Canada has been changing. Major peer-reviewed funding from grant agencies is highly competitive, with success rates typically less than $20 \%$. There is an increasing trend towards targeted research priorities and an expectation that researchers are working in collaborative, often interdisciplinary teams to optimize inter-institutional expertise and resources. Research activity is subject to increasing regulatory complexity and accountability reporting. More importantly, research that may change practice often requires the involvement of many centres, not only to recruit sufficient subjects to evaluate clinically significant endpoints, but also to improve external validity and generalizability of findings.

The formation of the PACT initiative is in response to this environment, and it will advance perioperative anesthesiology research by providing a forum for developing a team approach to conducting investigations in unanswered clinical and basic science questions in anesthesiology, particularly where single centre trials may not be methodologically robust enough to answer these questions comprehensively.

Currently, there is limited anesthesiology representation on the Canadian Institutes of Health Research (CIHR) peer review committees. This is due, in part, to few clinician investigators in our specialty being involved in leadership roles in multicentre clinical research studies. Through its leadership, the PACT group can help to address this shortfall. Another potential benefit of the PACT group is the increased likelihood of funding success through a coordinated approach to grant applications. This success should lead to increased CIHR peer review committee membership and thus to an improved research profile for anesthesiology nationally.

Also, by helping to generate research successes, the PACT group could foster a new research culture within the entire anesthesiology community. Perhaps most importantly, anesthesiology residents will be exposed to the investigative process as part of their training, which might kindle an early interest in pursuing a research career.

The PACT programs benefit from peer review and from continuous collegial support through consultation with members who have experience in research program development, including questionnaire development, grant preparation, statistical expertise, proficiency with clinical trials methodology, management of large scale clinical trials, and manuscript preparation. This support is invaluable in the current research environment. Much can be achieved through a constructive and collaborative approach. Good projects become excellent, the feasibility of conducting a large scale randomized clinical trial can be determined, and, eventually, management of a multicentre clinical trial can be facilitated. The PACT group is available to support investigators in achieving their goals. However, the PACT group is not a funding agency; it provides an environment where ideas can be generated and developed in a collegial atmosphere. The PACT group will provide peer review of grants when applications are ready for submission, and will assess protocols and publications prior to submission - indeed this is a requirement for any PACT-sponsored research program, and investigators commit to this process when they agree to have the project become a PACT-sponsored program.

\section{How will the PACT initiative work, and how is it organized?}

The PACT initiative will be based on the framework of the Canadian Critical Care Trials Group (CCCTG), an extremely effective collaborative research network in critical care. The CCCTG functions as a collaborative network of investigators in critical care medicine from across the country. In this group, research programs are developed and implemented based on the interest and expertise of members within the group, which includes methodologists with expertise in basic science, systematic reviews and metaanalysis, questionnaire development, and clinical trials development and implementation. Very little support for infrastructure is provided by CCCTG; most assistance is provided by in-kind support at the local institutions and from members, while all research is conducted through peer-reviewed grant funding. The group does not participate in industry-sponsored initiatives, although industry support for investigator-led, investigator-initiated projects may be considered. It is the most successful clinical trials network in critical care in the world, and its model is being replicated by other critical care trials networks internationally (e.g., Australia and Scotland) and by other medical specialities.

The PACT group seeks to emulate the CCCTG model as it reflects the realities of the current funding situation in Canada. In the past, the major funding institution for clinical research in Canada, Canadian Institutes of Health Research (CIHR), has not provided much in the way of infrastructure support. Moreover, by having a broad-based multicentred approach to funding with support provided locally within each participating centre, there is less risk for failure of the entire research program should funding within a single centre be diminished or suspended for any reason.

The Figure provides an overview of the organizational structure of the PACT group. The group functions through a Steering Committee, which is the governance committee that makes policy decisions and provides scientific and 


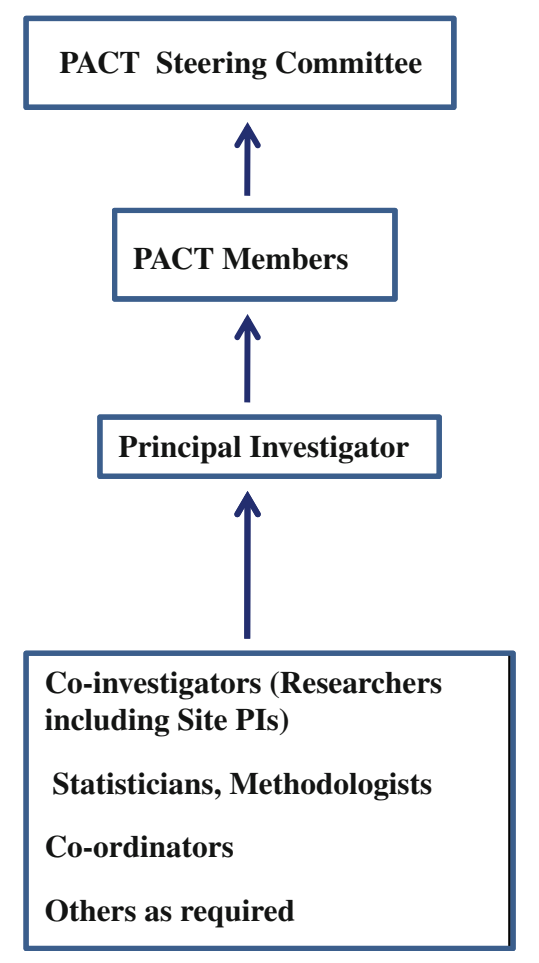

Figure Organizational structure of The Canadian Perioperative Anesthesiology Clinical Trials Group

administrative support to the membership. The Steering Committee will review all protocols prior to the PACT meeting, prior to grant submission, and prior to submission of manuscripts for publication. Written feedback will be provided within a timely period. A secretariat has been created within the Department of Anesthesiology at Dalhousie University to provide administrative support for the PACT group (contact canadianpact@ gmail.com) and a website is in development. A grant from the Association of Canadian University Departments of Anesthesiology (ACUDA) has provided start-up funding for the organization.

\section{How do I get the PACT group to consider my research idea?}

The success of the PACT initiative depends on the willingness of the academic community to produce research programs with the ultimate aim of conducting multicentre clinical trials to answer meaningful questions within the domain of anesthesiology and perioperative medicine. Of particular importance is the fact that this initiative is not restricted to any subspecialty area of interest. The process for submitting program proposals to the PACT group for consideration will be as follows:

1. Investigators present their idea, background research, and initial proposal for investigation at a PACT group meeting.
2. Face-to-face collegial feedback is provided by the PACT members regarding the clinical relevance, methodology, and feasibility of the proposal.

3. Members are asked if they accept the proposal as a PACT-sponsored program of research.

4. If the proposal is accepted, the investigators agree to conduct their program of research in accordance with the guidance of the PACT group.

\section{Implementation and conclusions}

The success of the PACT initiative will largely depend on its acceptance and support by the Canadian anesthesiology research community. As previously stated, success of the initiative is ultimately defined by development of clinically relevant PACT-sponsored research programs, ultimate dissemination of the findings at international meetings, and publication in high impact journals. Clearly, given the model suggested, the larger the number of participating members the higher the likelihood of success. In terms of expertise, ideas, collaboration, etc., there is strength in numbers. The intent is to recruit anesthesiologists - both neophyte and experienced - who are interested in the design, conduct, and implementation of clinical trials within the broad context of the specialty, including perioperative medicine. Regardless of the stage in their respective careers, members can be assured that the PACT group will provide a supportive environment where members can feel welcome to contribute ideas and participate in developing their ideas into meaningful research questions and solutions. Providing a forum for such creative activity will enrich the investigative spirit within anesthesiology, promote professional development, improve the standards of conducting research, and ultimately enhance the standard of care. By encouraging national representation on the Steering Committee and intentionally scheduling meetings in different locales within Canada, the PACT group will encourage a broad representation from across the country. Please feel free to contact any member of the Steering Committee for additional information. Together we can make our PACT initiative a success.

\section{Peut-on établir un Groupe canadien d'essais cliniques périopératoires en anesthésiologie?}

La création d'un réseau de collaboration pour la recherche au Canada: qu'est-ce que le PACT?

Au Canada, les recherches menées dans le domaine de l'anesthésiologie se sont principalement limitées aux 
champs de la science fondamentale et de la physiologie appliquée. Bien qu'elles aient une pertinence et une importance scientifiques majeures, la plupart des ces recherches évaluent des résultats physiologiques et des résultats cliniques de substitution plutôt que des résultats significatifs d'un point de vue clinique, lesquels pourraient avoir des répercussions sur notre façon de pratiquer. Alors que nombre de spécialités médicales ont déjà réalisé l'importance de l'évaluation de résultats tels que la mortalité, la qualité de vie, la durée d'hospitalisation ou encore l'efficacité des soins, l'anesthésiologie est à la traîne. L'une des principales raisons qui expliquent ce retard de l'anesthésiologie au Canada dans l'adoption d'études de devenirs est liée à la mise en place tardive d'un réseau de collaboration entre chercheurs en anesthésie qui aura pour objectif de faire avancer les projets de recherche clinique multicentriques. La recherche clinique se trouve aujourd'hui à un tournant et évolue rapidement - non seulement au Canada, mais partout dans le monde. Nous sommes convaincus que les anesthésiologistes canadiens peuvent améliorer de façon notable leurs capacités de recherche en promouvant les recherches en collaboration s'intéressant aux devenirs. Nous pensons également que de telles recherches ont le potentiel de changer - et changeront - la pratique clinique. Afin d'atteindre cet objectif, nous avons créé un réseau de collaboration pour la recherche, le groupe d'essais cliniques périopératoires en anesthésiologie, ou PACT (Perioperative Anesthesiology Clinical Trials).

Le groupe PACT est un groupe participatif formé d'anesthésiologistes universitaires canadiens s'intéressant à la conception, à la mise en œuvre, à la réalisation et à la publication d'essais cliniques multicentriques dans les domaines de l'anesthésiologie et de la médecine périopératoire. Le but de l'initiative PACT est d'aider les chercheurs cliniciens en anesthésiologie à: 1) identifier et explorer des questions pertinentes d'un point de vue clinique en anesthésiologie et en médecine périopératoire; 2) mettre en place des programmes de recherche qui répondent à leurs questions de recherche; 3) structurer et réaliser des recherches de qualité basées sur une méthodologie rigoureuse; 4) faciliter la collaboration entre divers chercheurs partout au pays afin de réaliser des recherches multicentriques; 5) obtenir du financement fondé sur l'évaluation par les pairs de la part d'organismes subventionnaires; et finalement 6) réaliser et publier leurs résultats dans des revues à impact élevé dotées de comités de pairs. Le but premier de l'initiative PACT est de générer des connaissances en anesthésie périopératoire en créant un forum qui favorise l'élaboration et la mise en œuvre d'études cliniques multicentriques conçues pour répondre aux questions de recherche. Nous prévoyons que ce forum aura un impact fondamental sur la pratique de l'anesthésiologie et de la médecine périopératoire. De plus, en tant que réseau participatif, le groupe PACT proposera un système de mentorat aux nouveaux chercheurs et favorisera la diffusion des connaissances de recherche, des meilleures pratiques ainsi que la standardisation des paramètres de pratique. Ainsi, le PACT contribuera à la pratique de l'anesthésiologie et garantira que la profession progresse continuellement.

\section{Pourquoi avons-nous besoin de l'initiative PACT, et quels sont les avantages si mon programme devient un programme de recherche commandité par le PACT?}

L'environnement de la recherche au Canada a évolué. La concurrence pour l'obtention de subventions importantes accordées suite à l'évaluation par les pairs par les organismes subventionnaires est féroce, les taux de réussite se situant typiquement au-dessous de $20 \%$. La tendance est de plus en plus aux priorités de recherche ciblées, et on attend des chercheurs qu'ils travaillent en collaboration, souvent au sein d'équipes interdisciplinaires, afin d'optimiser l'expertise et les ressources de plusieurs institutions. Les activités de recherche doivent respecter des règles et des normes de déclaration d'imputabilité de plus en plus complexes. Plus important encore, les recherches qui pourraient avoir un impact sur la pratique nécessitent souvent la participation de plusieurs centres, non seulement pour recruter suffisamment de patients afin d'évaluer des résultats significatifs d'un point de vue statistique, mais également pour en améliorer la validité externe et l'aspect généralisable.

L'initiative PACT a été créée en réponse à cet environnement, et elle fera progresser la recherche en anesthésiologie périopératoire en offrant un forum qui permettra de tirer parti d'une approche d'équipe pour la réalisation d'études portant sur des questions cliniques et de science fondamentale en anesthésiologie auxquelles aucune réponse n'a encore été apportée, particulièrement dans les cas où des études réalisées dans une seule institution pourraient ne pas être suffisamment robustes pour répondre à ces questions de façon exhaustive.

À l'heure actuelle, la représentation de notre profession dans les comités de pairs des Instituts de recherche en santé du Canada (IRSC) est encore limitée. Cet état de fait est dû en partie au nombre restreint de chercheurs cliniciens de notre spécialité qui occupent des fonctions de leadership dans les études multicentriques de recherche clinique. Grâce à son leadership, le groupe PACT pourra pallier ce manque. Un autre avantage potentiel du groupe PACT est la probabilité accrue de recevoir du financement grâce à une approche coordonnée des soumissions de candidature pour les subventions. L'obtention de telles subventions devrait générer une participation accrue de notre spécialité 
aux comités d'évaluation par les pairs des IRSC - et, par conséquent, améliorer le profil de recherche de l'anesthésiologie au niveau national.

De plus, en procurant son assistance pour le succès des recherches, le groupe PACT pourrait favoriser l'apparition d'une nouvelle culture de recherche au sein de toute la communauté de l'anesthésiologie. Mais, plus important peut-être, les résidents en anesthésiologie seront exposés au processus de recherche dans le cadre de leur formation, ce qui pourrait susciter un intérêt précoce dans une carrière de recherche.

Les programmes du PACT bénéficient d'une évaluation par les pairs et d'un soutien collégial constant grâce à un processus de consultation avec des personnes ayant de l'expérience dans la mise au point de programmes de recherche, notamment dans l'élaboration de questionnaires, la préparation des demandes de subventions, l'expertise statistique, la connaissance de la méthodologie des études cliniques, la gestion d'études cliniques à grande échelle et la préparation de manuscrits. Ce soutien est inestimable dans l'environnement de recherche actuel. D'importants résultats peuvent être atteints grâce à une approche constructive et collaborative. Les bons projets deviennent excellents, la faisabilité d'une étude clinique randomisée à grande échelle peut être déterminée et, en fin de compte, la gestion d'une étude clinique multicentrique peut être facilitée. La raison d'être du groupe PACT est de soutenir les chercheurs dans l'atteinte de leurs objectifs. Toutefois, le groupe PACT n'est pas un organisme subventionnaire; il offre un environnement dans lequel des idées peuvent être générées et élaborées dans une ambiance collégiale. Le groupe PACT proposera une révision par les pairs des candidatures pour subventions lorsque celles-ci seront prêtes à être soumises, et il évaluera les protocoles et les publications avant leur soumission - il s'agit là d'une exigence pour tout programme de recherche soutenu par le PACT; les chercheurs acceptent ce processus lorsqu'ils consentent à ce que leur projet devienne un programme soutenu par le PACT.

\section{Comment l'initiative PACT fonctionnera-t-elle et comment est-elle organisée?}

L'initiative PACT s'inspirera de la structure du groupe CCCTG (Canadian Critical Care Trials Group), un réseau extrêmement efficace de collaboration de recherche en soins critiques. Le CCCTG fonctionne comme un réseau de collaboration entre les chercheurs en médecine des soins critiques provenant des quatre coins du pays. Dans ce groupe, les programmes de recherche sont élaborés et mis en œuvre selon l'intérêt et l'expertise des membres, parmi lesquels on compte des spécialistes de la méthodologie proposant une expertise dans les domaines suivants: science fondamentale, comptes-rendus méthodiques et méta-analyse, élaboration de questionnaires, ainsi que mise au point et mise en œuvre d'études cliniques. Le CCCTG ne fournit qu'un soutien minimal au niveau des infrastructures; la plupart de l'aide aux chercheurs se fait par le biais d'un soutien concret dans les institutions locales et de la part de membres, alors que toutes les recherches sont réalisées à l'aide d'un financement évalué par les pairs. Le groupe ne participe pas aux initiatives financées par l'industrie, bien que le soutien de l'industrie puisse être pris en compte pour les projets menés et initiés par un chercheur. Il s'agit du réseau d'études cliniques le plus réussi en matière de soins critiques au monde, et son modèle est reproduit aux quatre coins de la planète par d'autres réseaux d'études cliniques en soins critiques (par ex. en Australie et en Écosse) ainsi que dans d'autres spécialités.

Le groupe PACT souhaite imiter le modèle du CCCTG étant donné qu'il reflète les réalités de la situation actuelle du financement au Canada. Par le passé, l'organisme subventionnaire le plus important au Canada pour la recherche clinique, soit les Instituts de recherche en santé du Canada (IRSC), n'a jamais fourni beaucoup de soutien au niveau des infrastructures. De plus, en adoptant une approche multicentrique et globale du financement, accompagnée du soutien accordé au niveau local au sein de chaque centre participant, le risque d'échec total d'un programme de recherche est plus faible - même si le financement dans un centre unique devait être suspendu ou réduit pour quelque raison que ce soit.

Le diagramme ci-joint résume la structure organisationnelle du groupe PACT. Le groupe fonctionne à l'aide d'un Comité directeur, qui est le comité de gouvernance qui prend les décisions en matière de politiques et fournit un soutien scientifique et administratif aux membres. Le Comité directeur passera en revue tous les protocoles avant la réunion du PACT, avant la soumission pour subvention, et avant la soumission des manuscrits pour publication. Des commentaires écrits seront adressés au chercheur dans un délai acceptable. Un secrétariat a été créé au sein du département d'anesthésiologie de l'Université Dalhousie pour servir de soutien administratif au groupe PACT (contact: canadianpact@gmail.com) et un site Internet est en cours d'élaboration. Une bourse de l'Association canadienne des départements universitaires d'anesthésie (ACUDA) a versé les fonds nécessaires à la création de l'organisme.

\section{Comment dois-je procéder pour que le groupe PACT évalue mon idée de recherche?}

Le succès de l'initiative PACT repose sur la volonté de la communauté universitaire à créer des programmes de 


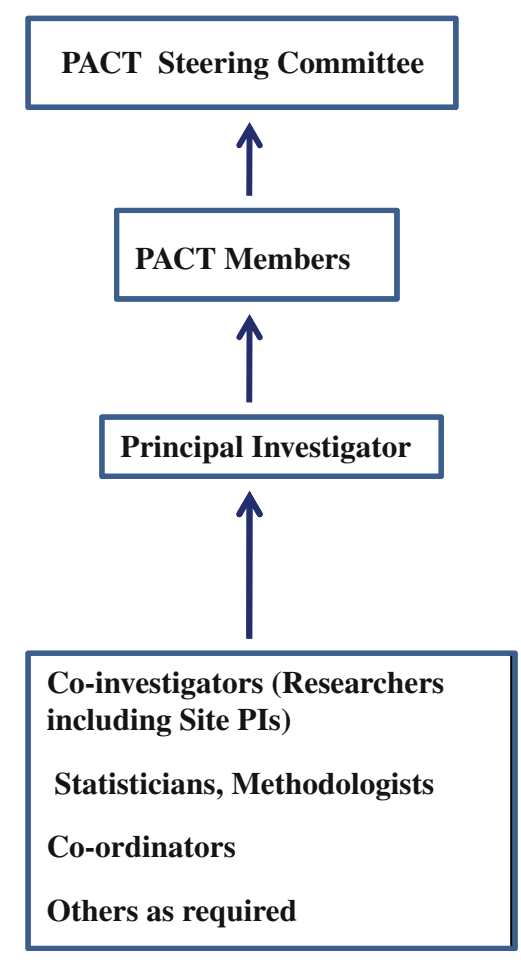

Figure Structure organisationnelle du Groupe canadien d'essais cliniques en anesthésiologie périopératoire (PACT)

recherche dont l'objectif final est de réaliser des études cliniques multicentriques qui répondent à des questions importantes touchant aux domaines de l'anesthésiologie et de la médecine périopératoire. Il convient de noter que cette initiative ne se limite pas à une sous-spécialité en particulier. Le processus de soumission de propositions de programme au groupe PACT sera le suivant:

1. Les chercheurs présentent leur idée, leurs recherches préliminaires et une proposition initiale de recherche lors d'une réunion du groupe PACT.

2. Une rétroaction collégiale en face à face est offerte par les membres du PACT quant à la pertinence clinique, la méthodologie et la faisabilité de la proposition.

3. On demande aux membres s'ils acceptent la proposition en tant que programme de recherche soutenu par le PACT.

4. Si la proposition est acceptée, les chercheurs s'engagent à réaliser leur programme de recherche conformément aux directives du groupe PACT.

\section{Mise en œuvre et conclusion}

Le succès de l'initiative PACT reposera en grande partie sur l'accueil et le soutien dont elle fera l'objet au sein de la communauté canadienne de recherche en anesthésiologie.
Comme cela a été précédemment mentionné, le succès de l'initiative sera en fin de compte déterminé par l'élaboration de programmes de recherche soutenus par le PACT et pertinents d'un point de vue clinique, la diffusion finale des résultats lors de congrès internationaux, et la publication dans des revues à fort impact. Au vu du modèle suggéré, il est clair que plus le nombre de participants sera élevé, plus il est probable que l'initiative réussisse. En termes d'expertise, d'idées, de collaboration, etc. - l'union fait la force. Notre intention est de recruter des anesthésiologistes, à tous les stades de leur carrière, qui sont intéressés par la conception, la réalisation et la mise en œuvre d'études cliniques dans le contexte étendu de notre spécialité, y compris la médecine périopératoire. Nous garantissons aux membres que le groupe PACT fournira un environnement de soutien où leurs idées seront les bienvenues et dans lequel ils pourront participer à la transformation de leurs idées en questions et solutions de recherche importantes et ce, indépendamment du stade de leur carrière. La création d'un forum encourageant une activité créatrice d'une telle envergure enrichira sans aucun doute l'esprit de recherche au sein de l'anesthésiologie, favorisera le développement professionnel, améliorera les normes pour effectuer des recherches et fera progresser, en fin de compte, les normes de soins. En encourageant une représentation nationale dans le Comité directeur et en organisant intentionnellement les réunions dans différents sites au Canada, le groupe PACT souhaite favoriser une vaste représentation provenant de partout au pays. Pour toute information supplémentaire, nous vous invitons à contacter un membre du Comité directeur. Ensemble, nous créerons le succès de l'initiative PACT.

Competing interests Drs. Hall and Turgeon are members of the Canadian Critical Care Trials Group. Drs. Hall, Beattie, and Yang are members of the CANNECTIN Clinical Trials research group.

\section{APPENDIX}

Perioperative Anesthesiology Clinical Trials (PACT) Group Steering Committee

Dr. Richard Hall - Chair

Professor of Anesthesiology, Medicine and

Pharmacology,

Associate Professor of Surgery,

Chair, Cardiovascular Anesthesia Research

Dalhousie University

E-mail: rihall@dal.ca

Dr. Scott Beattie

Professor of Anesthesiology

R. Fraser Elliot Chair in Cardiac Anesthesia 
Deputy Anesthetist in Chief

Director of Clinical Research

University Health Network

University of Toronto

E-mail: scott.beattie@uhn.on.ca

Dr. Davy Cheng

Distinguished University Professor

Chair /Chief, Department of Anesthesia \& Perioperative

Medicine

London Health Sciences Centre \& St. Joseph Health

Care

University of Western Ontario

E-mail: Davy.Cheng@1hsc.on.ca

Dr. Peter Choi

Associate Professor \& Clinical Research Director

(Anesthesiology)

Department of Anesthesiology, Pharmacology \&

Therapeutics

The University of British Columbia

E-mail: Peter.Choi2@vch.ca

Dr. André Y. Denault

Professeur agrégé de clinique or Associate Professor

Département d'Anesthésiologie

Affilié au département de Médecine,

Division des soins intensifs du Centre Hospitalier

de l'Université de Montréal

E-mail: andre.denault@gmail.com
Dr. David Mazer

Professor of Anesthesia and Physiology

Department of Anesthesia

University of Toronto

E-mail: mazerd@smh.ca

Dr. W. Alan C. Mutch

Professor

Vice Chair Research and Academic Affairs

Department of Anesthesia

University of Manitoba

E-mail: wacmutch@shaw.ca

Dr. Alexis F. Turgeon

Assistant Professor

Research Director

Department of Anesthesiology,

Division of Critical Care Medicine,

Université Laval

E-mail: Alexis.Turgeon@fmed.ulaval.ca

Dr. Homer Yang

Professor of Anesthesiology

Chair \& Chief

Department of Anesthesia

University of Ottawa

E-mail: hyang@ottawahospital.on.ca 